\title{
Prevalence of congenital coronary artery anomalies and variants in 726 consecutive patients based on 64-slice coronary computed tomography angiography
}

\author{
K. Szymczyk ${ }^{1}$, M. Polguj², E. Szymczyk³ ${ }^{3}$ A. Majos ${ }^{1}$, P. Grzelak¹, L. Stefańczyk ${ }^{1}$ \\ ${ }^{1}$ Department of Radiology, Barlicki University Hospital, Medical University of Lodz, Poland \\ 2Department of Angiology, Chair of Anatomy, Medical University of Lodz, Poland \\ ${ }^{3}$ Department of Cardiology, Medical University of Lodz, Poland
}

[Received 18 June 2013; Accepted 24 July 2013]

Background: Coronary computed tomography angiography (CCTA) is helpful in making a precise noninvasive evaluation of coronary anatomy, allowing concomitant evaluation of other cardiac structures. The aim of this study was to determine the prevalence of coronary artery variations detected by 64-slice mutidetector CT. Materials and methods: The results of ECG-gated CCTA in 726 consecutive patients (mean age 58 years) were analysed retrospectively. The main indications for CCTA were atypical chest pain, angina pectoris, screening for coronary artery disease and determination of the patency of bypass grafts or stents. Acquisition was performed with a 64-detector CT scanner with retrospective ECG gating. Imaging results were assessed by experienced cardiovascular radiologist.

Results: The overall incidence of coronary artery anomalies was 1.1\% (8 out of 726 participants). The most common anomaly was an anomalous origin of the circumflex artery from the right coronary sinus with a retroaortic course (4 patients, $0.6 \%)$, followed by origin of right coronary artery from the left coronary sinus (2 patients, $0.3 \%$ ). One patient with abnormal origin of the left main artery from the right coronary sinus (0.1\%) and 1 patient with a circumflex artery origin from the proximal segment of the right coronary artery $(0.1 \%)$ were observed, both with retroartic course.

Conclusions: CCTA is a noninvasive imaging technique useful for the precise evaluation of variations of the coronary arteries. This study shows similar results to other reports on this subject. (Folia Morphol 2014; 73, 1: 51-57)

Key words: coronary artery, anatomical variations, multidetector cardiac computed tomography

\section{INTRODUCTION}

The term, coronary artery anomaly refers to anatomical variations of the coronary artery that are present in a small percentage of the population [11]. The reported prevalence ranges from $0.7 \%$ to $2.4 \%$ in a cardiac imaging series $[24,25]$. In otherwise nor- mal patients, there may be variations in the number, shape, and location of the ostia or origins of the coronary arteries [16]. Most of these variations appear to be of no clinical significance, although some patients will present for medical attention with a serious first presentation, such as myocardial infarction or sudden 
cardiac death (SCD) [9]. Certain types of coronary artery anomalies have been noted to confer a higher risk of $S C D$, these being of mainly nonaortic origin or those with an origin from the opposite coronary sinus and an interarterial course [23]. For many years, the diagnosis of coronary artery anomalies was limited to postmortem autopsy or invasive coronary angiography. Currently, coronary computed tomography angiography (CCTA) is the main alternative to invasive angiography in the evaluation of coronary anatomy, allowing concomitant evaluation of other cardiac structures [7].

The aim of this study was to determine the prevalence of coronary artery variations detected by 64 -slice mutidetector CT.

\section{MATERIALS AND METHODS}

\section{Patients}

The results of ECG-gated CCTA examinations performed on 726 consecutive patients were analysed retrospectively. All examinations were performed at Barlicki University Hospital (Medical University of Lodz, Poland) from December 2010 to May 2013. The mean age of the patients was 58 years, $53 \%$ were male. The main indications for CCTA were atypical chest pain, angina pectoris, screening for coronary artery disease and determination of the patency of bypass grafts or stents. The exclusion criteria for CCTA were arrhythmia (atrial fibrillation, frequent ventricular and supraventricular premature beats), high coronary artery calcium score (CACS) result (> $800 \mathrm{AU}$ ), previous serious allergic reaction to iodine contrast medium, renal failure and pregnancy.

\section{Computed tomography protocols and image reconstruction}

All studies were performed using a 64-detector CT scanner with retrospective ECG gating (Lightspeed VCT, GE Healthcare, Milwaukee, WI, USA). Firstly, the CACS was determined in all patients. If CACS was over $800 \mathrm{AU}$, then CCTA was not performed. In the remaining patients, complete $\mathrm{CT}$ evaluation was performed with the administration of a contrast agent. The heart rate of patients ranged between 52 and $97 \mathrm{bpm}$ (mean $64 \mathrm{bpm}$ ); intravenous beta-blocker (up to a total dose of $20 \mathrm{mg}$ metoprolol) was used just before the scan if the heart rate was greater than $70 \mathrm{bpm}$, as long as no contraindication was present.
An intravenous cannula was inserted in the right basilica vein. The triphasic injection protocol was used with an injection of $60-70 \mathrm{~mL}$ of non-ionic contrast medium with high iodine concentration (iomeprol $400 \mathrm{mgl} / \mathrm{mL}$, lomeron) at a flow rate of $4.8-5.2 \mathrm{~mL} / \mathrm{s}$. This was followed by injection of a $40 \mathrm{~mL}$ solution of $20 \%$ contrast medium and $80 \%$ saline solution, and finally an injection of $30 \mathrm{~mL}$ saline with the same flow rate as used for CCTA.

The CCTA data was analysed on cardiac dedicated workstation (Advantage Workstation 4.4, GE Healthcare, Milwaukee, WI, USA) using curved multiplanar reconstruction (MPR) and 3-dimensional volume rendering reconstructions. Images were evaluated by an experienced cardiovascular radiologist. The diameters of the coronary arteries were evaluated at a distance 3 to $5 \mathrm{~mm}$ from the origin of the vessels. If the cross section of the artery was not round in shape, two perpendicular measurements were taken and mean value was obtained.

\section{Definitions}

The dominant coronary artery was defined on the basis of which coronary artery passed gave off the posterior descending artery. If the posterior descending artery stemmed from the right coronary artery and the posterolateral artery stemmed from the left circumflex artery, then the vasculature of the heart was classified as co-dominant. Coronary artery anomalies were recognised according to the classification proposed by Angelini [2], which divides coronary artery anomalies into 3 categories: anomalies of origination and course, anomalies of intrinsic coronary arterial anatomy, and anomalies of coronary termination.

A coronary artery aneurysm is defined as the condition in which coronary artery segments have a diameter more than 1.5 times greater than that of the diameter of the normal adjacent coronary segments or the diameter of the patient's largest coronary vessel and involve less than $50 \%$ of the total length of the vessel [17]. The term "ectasia" was used to describe a condition in which diffuse dilatation over 1.5 times than the normal diameter of the coronary arteries occurs, which involves $50 \%$ or more of the length of the artery [17].

Myocardial bridging was defined as an intra-myocardial segment in the course of a major epicardial coronary artery [14].

\section{RESULTS}

Coronary artery circulation was right dominant in $580(79.9 \%)$ patients, left dominant in 

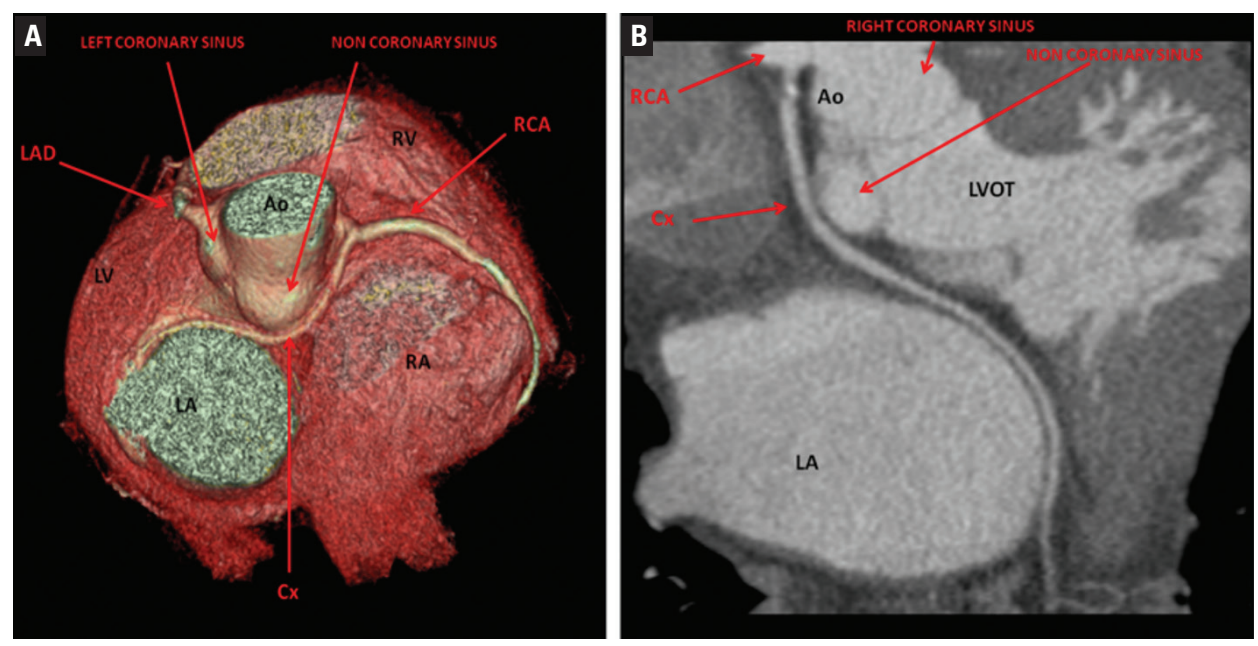

Figure 1. Variant of the anomalous origins of the circumflex artery from the right coronary artery with a retroaortic course; $\mathbf{A}$. Volume rendering, posterior view, right and left atrial appendages were removed to visualise coronary arteries; B. Mulitiplanar curved reconstruction in the plane of circumflex artery; Ao — aorta; Cx — circumflex artery; LA — left atrium; LAD — left anterior descending artery; LV — left ventricle; LVOT — left ventricular outflow tract; RA — right atrium; RCA — right coronary artery; RV — right ventricle.

$81(11.1 \%)$ patients, and co-dominant in $65(9.0 \%)$ patients. The left main coronary artery trifurcated into the intermediate artery in $246(33.9 \%)$ patients. The conus artery originated from the right coronary artery in 566 (77.9\%) patients and had a separate direct origin from the right coronary sinus in 160 (22.0\%) patients. A myocardial bridge was the most frequent coronary variant observed in $161(22.2 \%)$ patients, while coronary ectasia and aneurysm were diagnosed in $5(0.7 \%)$ patients and $2(0.3 \%)$ patients, respectively.

The overall incidence of coronary artery anomalies was $1.1 \%$ ( 8 out of 726 participants). The most common anomaly was an anomalous origin of the circumflex artery from the right coronary sinus with a retroaortic course (4 patients, $0.6 \%$ ) (Fig. 1), followed by the presence of the origin of right coronary artery from the left coronary sinus (2 patients, $0.3 \%$ ). In the first case, signs of compression were found in the interarterial course between the pulmonary trunk and the aorta, which is considered a "malignant variant" (Fig. 2), while the second patient had a similar, but more inferior (subpulmonic) course of the right coronary artery between the right ventricular outflow tract and the root of the aorta with no arterial compression and no clinical significance (Fig. 3). One patient was observed with an abnormal origin of the left main artery from the right coronary sinus (0.1\%) (Fig. 4) and another patient with a circumflex artery origin from proximal segment of right coronary artery $(0.1 \%)$, although both cases demonstrated a retroartic course. In patients with coronary anomalies, no other concomitant congenital heart diseases were observed.

The dominant vascularity type was analysed in the subgroup of patients with diagnosed coronary arterial anomalies. When the right coronary artery originated from left coronary sinus, the circumflex and right coronary arteries were noted as being co-dominant. Among patients with the origin of the circumflex artery stemming from the right coronary sinus, the circumflex artery was observed to be dominant only in 1 case: the right coronary artery was dominant in the rest of the patients. In both patients with the left main artery originating from the right coronary sinus and with the circumflex artery originating from the right coronary artery, the right coronary artery was dominant. The distribution of vascularity type in patients with an anomalous origin of the coronary artery and its diameters is presented in Table 1.

\section{DISCUSSION}

Knowledge of the normal coronary artery anatomy and its common variations is crucial for the accurate assessment of CCTA [13]. Variations in coronary anatomy exist with respect to number, origin, distribution, position and structural relationship with other cardiac structures, and these must be taken into consideration in the evaluation of coronary artery anomalies [8]. They may be isolated findings or 

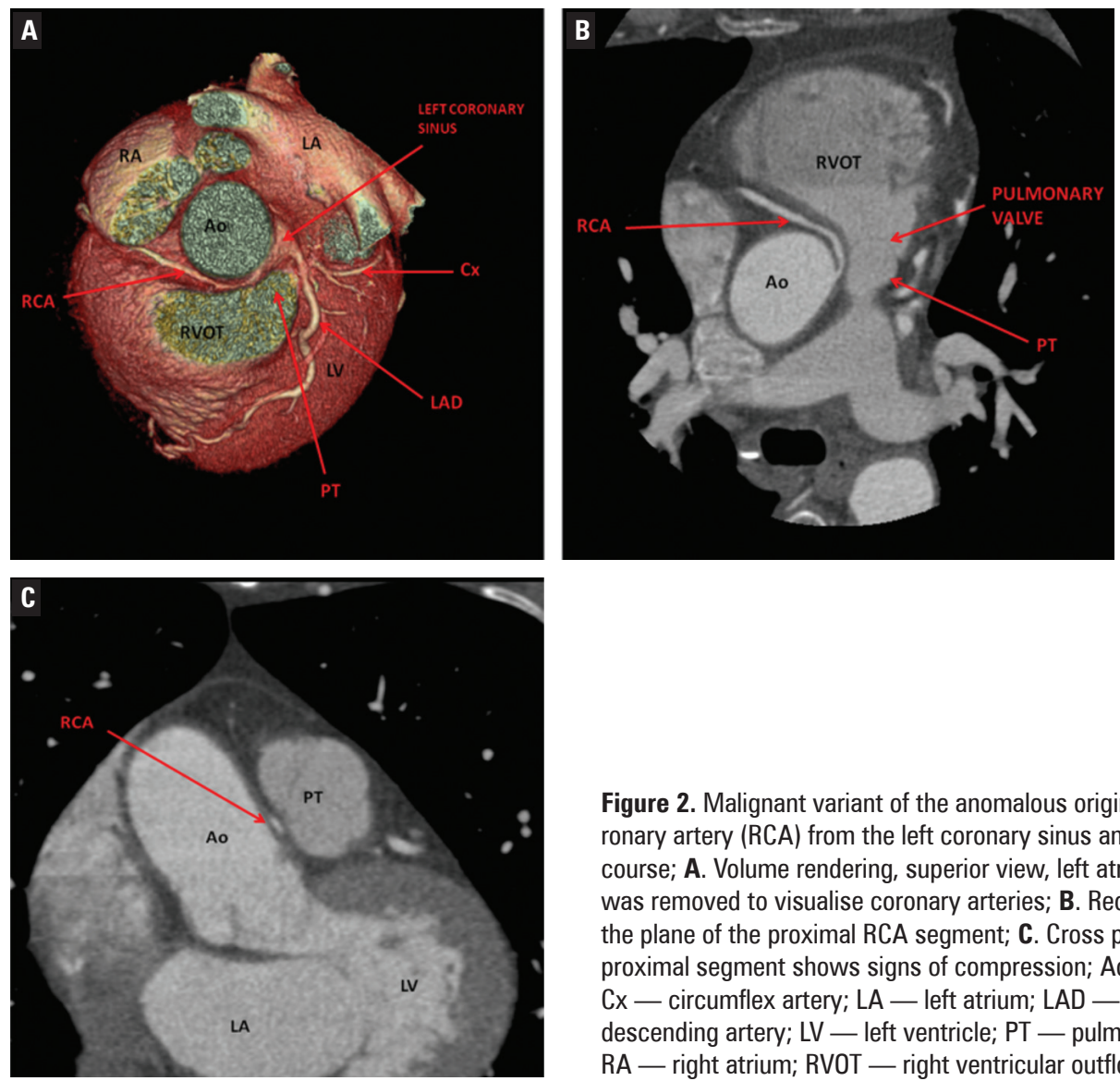

Figure 2. Malignant variant of the anomalous origin of the right coronary artery (RCA) from the left coronary sinus and an interarterial course; A. Volume rendering, superior view, left atrial appendage was removed to visualise coronary arteries; $\mathbf{B}$. Reconstruction in the plane of the proximal RCA segment; $\mathbf{C}$. Cross plane of the RCA proximal segment shows signs of compression; Ao - aorta; Cx — circumflex artery; LA — left atrium; LAD — left anterior descending artery; LV — left ventricle; PT — pulmonary trunk; RA — right atrium; RVOT — right ventricular outflow tract.
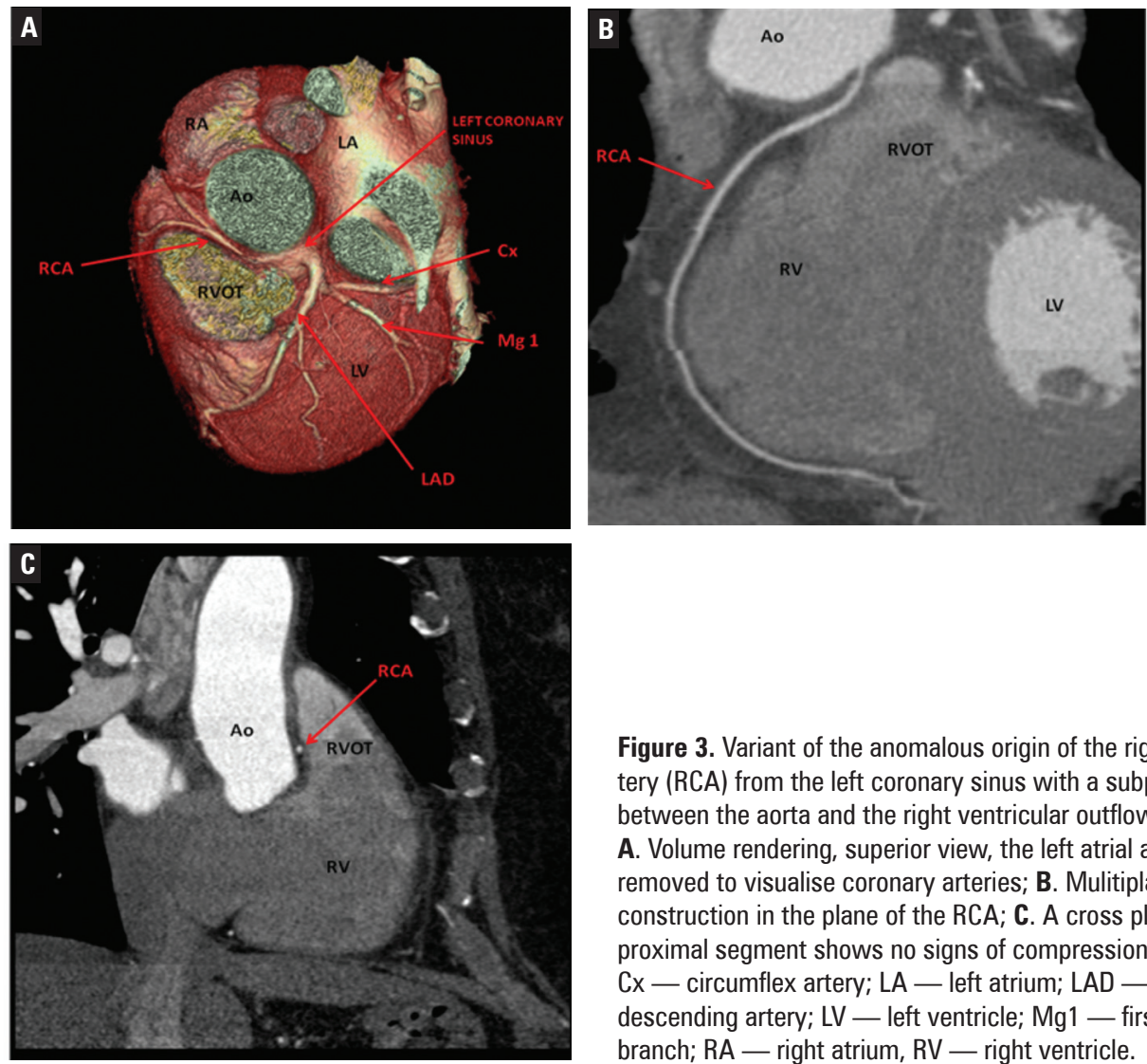

Figure 3. Variant of the anomalous origin of the right coronary artery (RCA) from the left coronary sinus with a subpulmonic course between the aorta and the right ventricular outflow tract (RVOT);

A. Volume rendering, superior view, the left atrial appendage was removed to visualise coronary arteries; B. Mulitiplanar curved reconstruction in the plane of the RCA; C. A cross plane of the RCA proximal segment shows no signs of compression; $\mathrm{Ao}$ - aorta; Cx — circumflex artery; LA — left atrium; LAD — left anterior descending artery; LV — left ventricle; Mg1 — first marginal branch; RA — right atrium, RV — right ventricle. 


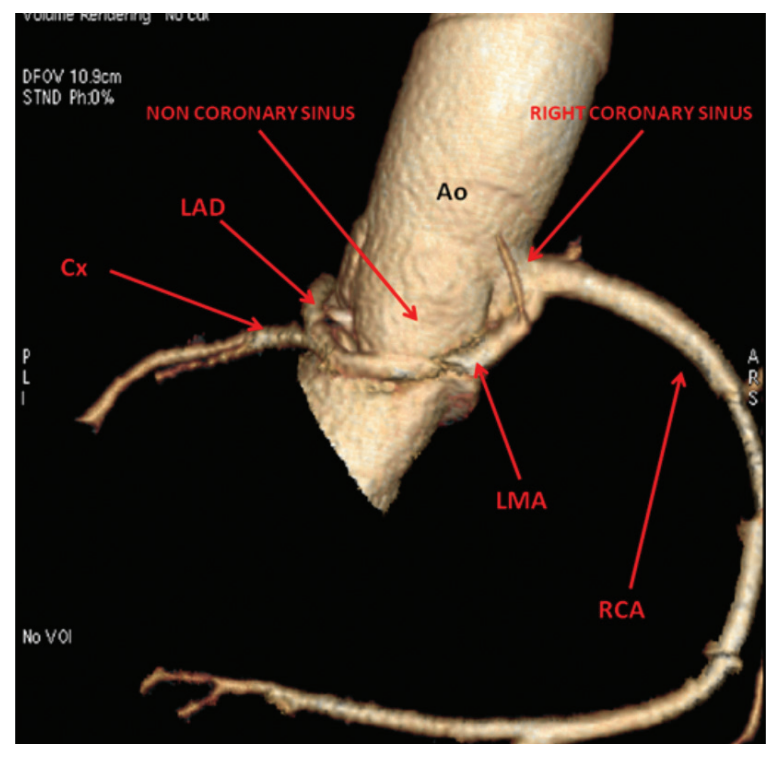

Figure 4. A variant of the anomalous origin of the left main artery from the right coronary sinus with a retroaortic course. A volume rendering of the aortic root and coronary arteries tree is presented; $\mathrm{Ao}$ - aorta; $\mathrm{Cx}$ - circumflex artery; LAD — left anterior descending artery; LMA — left main artery; RCA — right coronary artery.

coexist with other congenital heart conditions such as a bicuspid aortic valve, tetralogy of Fallot or transposition of the great arteries [19].

Most coronary artery anomalies are not of clinical importance, being asymptomatic and accidentally discovered on coronary angiography or autopsy. How- ever, some of them may be potentially life threatening. Among anomalies which can cause myocardial ischaemia and even sudden death are the presence of a coronary artery fistula, which is an anomalous origin of a coronary artery from the pulmonary trunk, or when the coronary artery stems from a contralateral side origin with an interarterial course between the aorta and the pulmonary artery [3].

While coronary artery anomalies are reported in approximately $1.3 \%$ of patients undergoing coronary angiography [24], the reported incidence of coronary artery anomalies diagnosed by CCTA is higher and ranges between $0.7 \%$ and $18 \%[4,22]$. These results are in accord with those of the present study: $1.0 \%$ of coronary artery anomalies being diagnosed in the study group.

Myocardial bridging was the most frequent normal variant observed in our study. The reported prevalence of myocardial bridging varies from $40 \%$ to $80 \%$ in autopsy studies and from $0.5 \%$ to $16 \%$ in conventional angiography [21]. The distinct difference between those 2 methods is due to the relatively low diagnostic sensitivity of conventional angiography in the visualisation of tunnelled segments of coronary arteries based only on systolic compression [12]. Cardiac computed tomography is an alternative method for the identification of myocardial bridging, which is of clinical importance due to its potential clinical consequences: ischaemia, acute coronary syndro-

Table 1. Summary of coronary artery anomalies, dominance types and arterial diameters in patients from the study group

\begin{tabular}{|c|c|c|c|c|c|c|c|}
\hline \multirow[t]{2}{*}{ Patient (initials) } & \multirow[t]{2}{*}{ Type of anomaly } & \multirow[t]{2}{*}{ Dominant artery } & \multicolumn{5}{|c|}{ Diameter of artery [mm] } \\
\hline & & & LMA & LAD & IM & CX & $\mathrm{RCA}$ \\
\hline UK & $\begin{array}{l}\text { RCA from LCS, } \\
\text { interarterial course }\end{array}$ & $\begin{array}{c}\text { Codominance } \mathrm{Cx} \\
\text { and RCA }\end{array}$ & 3.0 & 2.8 & - & 2.3 & 2.5 \\
\hline DB & $\begin{array}{l}\text { RCA from LCS, } \\
\text { subpulmonic course }\end{array}$ & $\begin{array}{c}\text { Codominance } \mathrm{Cx} \\
\text { and RCA }\end{array}$ & 4.2 & 3.0 & - & 2.8 & 2.0 \\
\hline MK & $\begin{array}{l}\text { LMA from RCS, } \\
\text { retroartic course }\end{array}$ & $\mathrm{RCA}$ & 3.7 & 3.0 & - & 1.6 & 4.5 \\
\hline TR & $\begin{array}{l}\text { Cx from } \mathrm{RCA}, \\
\text { retroartic course }\end{array}$ & $\mathrm{RCA}$ & 3.0 & 2.8 & 2.0 & 2.0 & 3.5 \\
\hline WH & $\begin{array}{l}\text { Cx from RCS, } \\
\text { retroartic course }\end{array}$ & $\mathrm{RCA}$ & - & 3.5 & - & 2.5 & 3.8 \\
\hline$J R$ & $\begin{array}{l}\text { Cx from RCS, } \\
\text { retroartic course }\end{array}$ & $C x$ & - & 3.1 & - & 3.0 & 1.8 \\
\hline TK & $\begin{array}{l}\text { Cx from RCS, } \\
\text { retroartic course }\end{array}$ & $\mathrm{RCA}$ & - & 3.0 & - & 2.5 & 4.0 \\
\hline $\mathrm{A} 0$ & $\begin{array}{l}\text { Cx from RCS, } \\
\text { retroartic course }\end{array}$ & $\mathrm{RCA}$ & - & 3.5 & 1.8 & 2.5 & 3.5 \\
\hline
\end{tabular}

CX — circumflex artery; IM — intermediate branch; LAD — left anterior descending artery; LCS — left coronary sinus; LMA — left main artery; RCA — right coronary artery; RCS - right coronary sinus 
me, coronary spasm, rupture of ventricular septum, arrhythmia or even SCD [1].

In contrary to 2-dimensional coronary angiography, CCTA is a non-invasive cardiovascular imaging method with a capability for multiplanar postprocessing and is able to show the origin, complex 3-dimensional anatomy, course and termination of the coronary arteries, making it the gold standard for visualisation of coronary artery anomalies [10, 26]. CCTA provides better images of the anatomic course of the coronary artery compared to invasive coronary angiography, enabling accurate demonstration of an anomalous or aberrant coronary vessel prior to operation or intervention [6, 20].

Even for experienced operators, the catheterisation of anomalous vessels may be a challenging and time-consuming procedure and additional diagnostics are often needed [5]. Schmid et al. [18] showed that CCTA was able to depict $100 \%$ of the anomalies diagnosed by conventional angiography. However, Shi et al. [21] revealed that conventional angiography failed to demonstrate $47 \%$ of the coronary anomalies which had been diagnosed by CCTA. Other imaging techniques seem to be still insufficient. The main limitation of echocardiography is the lack of an optimal acoustic window in a significant percentage of patients, while cardiac magnetic resonance is prone to cardiac and respiratory motion-related artefacts because of its low spatial resolution [15]. Therefore, CCTA seems to be the only current alternative to invasive coronary angiography in visualisation of coronary anomalies.

\section{CONCLUSIONS}

CCTA is a noninvasive imaging technique useful for the precise evaluation of variations and anomalies of the coronary arteries. Its 3-dimensional capabilities can clearly display the anatomy of coronary arteries. Further progress in temporal and spatial resolution with a concomitant reduction of radiation dose may overcome some current limitations and broaden the role of CCTA in coronary artery assessments. Being able to measure the diameters of presented arteries may be helpful in coronarography.

\section{REFERENCES}

1. Alegria JR, Herrmann J, Holmes DRJ, Lerman A, Charanjit $S$ (2005) Myocardial Bridging. Eur Heart J, 26: 1159-1168.
2. Angelini $P$ (2007) Coronary artery anomalies: an entity in search of an identity. Circulation, 115: 1296-1305.

3. Basso C, Maron BJ, Corrado D, Thiene G (2000) Clinical profile of congenital coronary artery anomalies with origin from the wrong aortic sinus leading to sudden death in young competitive athletes. J Am Coll Cardiol, 35: 1493-1501.

4. Cademartiri F, La Grutta L, Malagò R, Alberghina F, Meijboom WB, Pugliese F, Maffei E, Palumbo AA, Aldrovandi A, Fusaro M, Brambilla V, Coruzzi P, Midiri M, Mollet NR, Krestin GP (2008) Prevalence of anatomical variants and coronary anomalies in 543 consecutive patients studied with 64-slice CT coronary angiography. Eur Radiol, 18: 781-791.

5. de Jonge GJ, van Ooijen PM, Piers LH, Dikkers R, Tio RA, Willems TP, van den Heuvel AF, Zijlstra F, Oudkerk M (2008) Visualization of anomalous coronary arteries on dual-source computed tomography. Eur Radiol, 18: 2425-2432.

6. Erol C, Seker M (2011) Coronary artery anomalies: the prevalence of origination, course, and termination anomalies of coronary arteries detected by 64-detector computed tomography coronary angiography. J Comput Assist Tomogr, 35: 618-624.

7. Galas A, Hryniewiecki T, Kępka C, Michałowska I, Abramczuk E, Orłowska Baranowska E, Demkow M, Rużyłło W (2012) May dual-source computed tomography angiography replace invasive coronary angiography in the evaluation of patients referred for valvular disease surgery? Kardiol Pol, 70: 877-882.

8. Gawlikowska-Sroka A, Miklaszewska D, Czerwiński F (2010) Analysis of the influence of hesart size and gender on coronary circulation type. Folia Morphol, 69: 35-41.

9. Hill SF, Sheppard MN (2010) Non-atherosclerotic coronary artery disease associated with sudden cardiac death. Heart, 96: 1119-1125.

10. Jacobs JE (2010) Computed tomographic evaluation of the normal cardiac anatomy. Radiol Clin North Am, 48: 701-710.

11. Jappar IA, Chua T, Htoo MM, Cheah FK, Allen JC, Tan SY (2012) Diagnosis of anomalous origin and course of coronary arteries using non-contrast cardiac CT scan and detection features. J Cardiovasc Comput Tomogr, 6: 335-345.

12. Kim SY, Seo JB, Do KH, Heo JN, Lee JS, Song JW, Choe YH, Kim TH, Yong HS, Choi SI, Song KS, Lim TH (2006) Coronary artery anomalies: classification and ECG-gated multi-detector row CT findings with angiographic correlation. Radiographics, 26: 317-333.

13. Kini S, Bis KG, Weaver $L$ (2007) Normal and variant coronary arterial and venous anatomy on high-resolution CT angiography. Am J Roentgenol, 188: 1665-1674.

14. Ko SM (2008) An overview of myocardial bridging with a focus on multidetector CT coronary angiographic findings. Korean Circ J, 38: 583-589.

15. Montaudon $M$, Latrabe V, Iriart X, Caix P, Laurent F (2007) Congenital coronary arteries anomalies: review of the literature and multidetector computed tomography (MDCT)-appearance. Surg Radiol Anat, 29: 343-355.

16. Nowak D, Kozłowska H, Żurada A (2011) The relationship between the dimensions of the right coronary artery and the type of coronary vasculature in human foetuses. Folia Morphol, 70: 13-17. 
17. Pahlavan PS, Niroomand F (2006) Coronary artery aneurysm: a review. Clin Cardiol, 29: 439-443.

18. Schmid M, Achenbach S, Ludwig J, Baum U, Anders K, Pohle K, Daniel WG, Ropers D (2006) Visualization of coronary artery anomalies by contrastenhanced multi-detector row spiral computed tomography. Int J Cardiol, 111: 430-435.

19. Schmitt R, Froehner S, Brunn J, Wagner M, Brunner $H$, Cherevatyy O, Gietzen F, Christopoulos G, Kerber S, Fellner F (2005) Congenital anomalies of the coronary arteries: imaging with contrast-enhanced, multidetector computed tomography. Eur Radiol, 15: 1110-1121.

20. Serota H, Barth CW III, Seuc CA, Vandormael M, Aguirre F, Kern MJ (1990) Rapid identification of the course of anomalous coronary arteries in adults: the "dot and eye" method. Am J Cardiol, 65: 891-898.

21. Shi H, Aschoff AJ, Brambs HJ, Hoffmann MH (2004) Multislice CT imaging of anomalous coronary arteries. Eur Radiol, 14: 2172-2181.
22. Sundaram B, Kreml R, Patel S (2010) Imaging of coronary artery anomalies. Radiol Clin North Am, 48: 711$-727$.

23. Taylor AJ, Rogan KM, Virmani R (1992) Sudden cardiac death associated with isolated congenital coronary artery anomalies. J Am Coll Cardiol, 20: 640-647.

24. Yamanaka O, Hobbs RE (1990) Coronary artery anomalies in 126,595 patients undergoing coronary arteriography. Cathet Cardiovasc Diagn, 21: 28-40.

25. Yildiz A, Okcun B, Peker T, Arslan C, Olcay A, Bulent Va$\tan M$ (2010) Prevalence of coronary artery anomalies in 12,457 adult patients who underwent coronary angiography. Clin Cardiol, 33: E60-E64.

26. van Ooijen PM, Dorgelo J, Zijlstra F, Oudkerk M (2004) Detection, visualization and evaluation of anomalous coronary anatomy on 16-slice multidetector-row CT. Eur Radiol, 14: 2163-2171. 